\title{
Polarized Atomic Hydrogen Beam Studies in the Michigan Ultra-cold Jet ${ }^{1}$
}

\author{
R.S. Raymond*, B.B. Blinov*, N.S. Borisov ${ }^{\diamond}$, J. Cheng*, \\ A.M. Davidenko ${ }^{\triangle}$, V.V. Fimushkin ${ }^{\diamond}$, S.E. Gladycheva*, \\ V.N. Grishin ${ }^{\triangle}$, T. Kageya*, D.Yu. Kantsyrev*, D. Kleppner ${ }^{\sharp}$, \\ A.D. Krisch*, V.G. Luppov*, V.S. Morozov*, \\ J.R. Murray*, J.J. Neumann*, B. Yankama* \\ * Randall Laboratory of Physics, University of Michigan, Ann Arbor, MI 48109-1120 \\ $\diamond$ JINR, Dubna, Russia \\ $\triangle$ IHEP, Protvino, Russia \\ $\sharp$ Department of Physics, M.I.T., Cambridge, MA 02139
}

\begin{abstract}
Studies of an ultra-cold jet of polarized hydrogen atoms are described. This atomic beam is formed by the acceleration of cold $(0.3 \mathrm{~K})$ atoms emerging from a region of high magnetic field $(12 \mathrm{~T})$. The maximum measured density was about $10^{12}$ atoms $\mathrm{cm}^{-3}$. The beam's full width half maximum size was less than $4 \mathrm{~mm}$.
\end{abstract}

We are developing an ultra-cold jet of polarized hydrogen atoms to study spin effects in high energy collisions. The jet, in its test configuration, is shown in Fig. 1. Atoms from a room-temperature dissociator are cooled to $30 \mathrm{~K}$ and guided into a $12 \mathrm{~T}$ magnetic field by Teflon tubes. In this field, the atoms enter a separation cell in the mixing chamber of a dilution refrigerator and are cooled to $0.3 \mathrm{~K}$ by interactions with the superfluid- ${ }^{4} \mathrm{He}-$ coated cell walls. Atoms in the two lowerenergy hyperfine states are trapped in the high field, recombine, and are pumped away. Hydrogen atoms in the two upper hyperfine states accelerate out of the field to form an atomic beam. The separation cell's exit aperture is at $6 \mathrm{~T}$, which gives the beam an energy corresponding to about $4 \mathrm{~K}$; thus, its internal energy of $0.3 \mathrm{~K}$ makes the beam quite monochromatic.

Emerging from the cell, the cold atoms are first focused by a superfluid- ${ }^{4} \mathrm{He}-$ coated parabolic mirror; this reflection is mostly specular. The atoms are then further focused by an 11-cm bore sextupole magnet into a compression tube detector for absolute intensity measurements. The compression tube also allows measurements of the azimuthal and radial beam distributions. The maximum flux measured

1) This work is supported by the U.S. Department of Energy.

CP549, Intersections of Particle and Nuclear Physics: $7^{\text {th }}$ Conference, edited by Z. Parsa and W. J. Marciano (C) 2000 American Institute of Physics 1-56396-978-5/00/\$17.00 
so far is about $2.810^{15}$ atoms s $\mathrm{s}^{-1}$ into the compression tube's $12.5 \mathrm{~mm} \times 2.5 \mathrm{~mm}$ aperture. This corresponds to a density of about $10^{12}$ atoms $\mathrm{cm}^{-3}$, within the beam's measured full width half maximum size, which was less than $4 \mathrm{~mm}$.

We are also testing a maser, run in transient mode, to monitor the beam polarization. After injection of a power pulse, we integrate the free induction decay signal to obtain a number proportional to the difference in state populations of the two mixed states. By driving Zeeman transitions between the $\mathrm{J}=1$ hyperfine states before the maser transition, we learn about other state population differences. We have seen the main decay signal from the Jet's beam, but not the signatures of its Zeeman transitions. We are also developing a large-bore, cryogenic rf unit to drive transitions between the mixed hyperfine states, to enhance the final beam polarization. A room-temperature prototype $\mathrm{rf}$ unit has a measured transition efficiency of $97 \%$ with less than $150 \mathrm{~mW}$ of rf power. A new cold prototype rf cavity has a measured $\mathrm{Q}$ value of over 16,000 at $5 \mathrm{~K}$. We have also built and tested a $1.210^{7}$ liters $\mathrm{s}^{-1}$ cryocondensation pump for the jet's use in a storage ring.

Tests to improve the jet's beam intensity and operational characteristics continue.

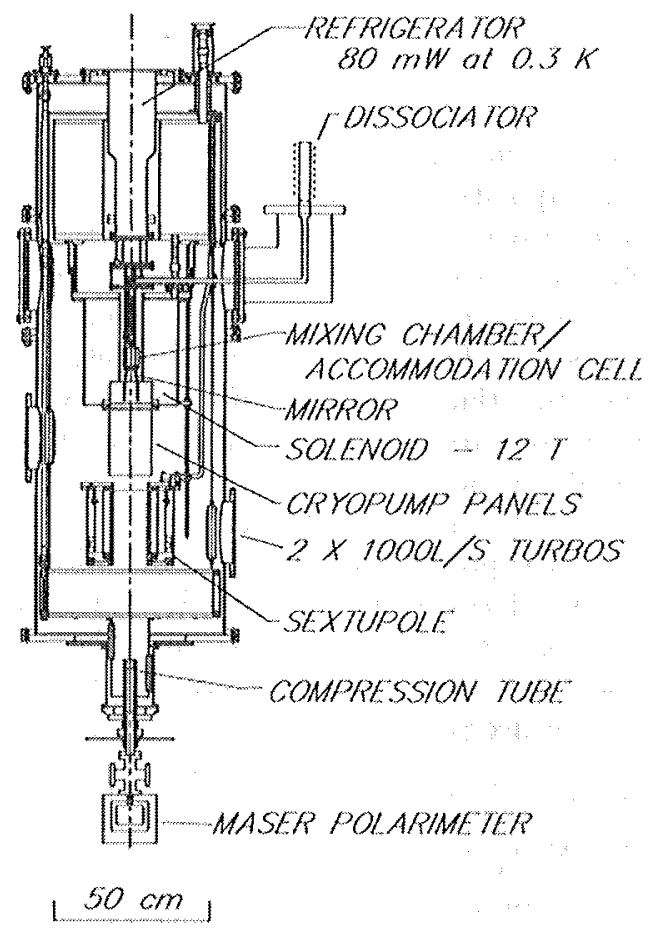

FIGURE 1. The test configuration of the Michigan ultra-cold polarized jet. 